\title{
The Contribution of the Amygdala to Conditioned Thalamic Arousal
}

\author{
Mary Eileen Cain, Bruce S. Kapp, and Corey B. Puryear \\ Department of Psychology, University of Vermont, Burlington, Vermont 05405
}

Previous research has demonstrated that thalamocortical neurons within the dorsal lateral geniculate nucleus (dLGN) are affected by an acoustic, fear-arousing, conditioned stimulus (Cain et al., 2000). This effect is reflected in an increase in activity and a tonic firing pattern, a pattern that assures the most accurate relay of information from the retina to the visual neocortex. Such an effect is considered to be indicative of a heightened state of arousal. The present research was designed to determine the extent to which the central nucleus of the amygdala (ACe) contributes to this effect. To this end, in experiment 1 extracellular recordings were made from single dLGN neurons in the awake rabbit during electrical stimulation of the ACe. Increased neuronal activity was observed in response to stimulation in the majority of neurons. Neurons that were in a burst firing pattern immediately before stimulation assumed a tonic firing pattern in response to stimulation. Experiment 2 was designed to determine whether inactivation of the ACe with muscimol would attenuate the response of dLGN neurons in the awake rabbit to the presentation of acoustic, fear-arousing, conditioned stimuli. Compared with vehicle injections, infusions of muscimol attenuated both the spontaneous activity and the response of dLGN neurons to the presentations of these stimuli. The results provide support for the hypothesis that the amygdala, and in particular the ACe, contributes to a heightened state of arousal during conditioned fear.

Key words: amygdaloid central nucleus; dorsal lateral geniculate nucleus; electrical stimulation; extracellular single unit recording; Pavlovian conditioning; muscimol
It has been proposed that fear elicits an enhanced state of arousal that influences the efficacy by which an organism detects and processes environmental stimuli (Kapp et al., 1992; Davis and Whalen, 2001). In support of this proposal are recent observations demonstrating that acoustic, fear-arousing, conditioned stimuli (CSs) affect thalamocortical neurons in the dorsal lateral geniculate nucleus (dLGN). These stimuli elicit increased firing in these neurons primarily because of a shift from a burst to a tonic firing mode (Cain et al., 2000). Tonic firing renders these neurons more sensitive to retinal input and is the most efficient mode for the transfer of information from the retina to the neocortex (Sherman, 1996; Sherman and Guillery, 2001). Tonic firing is predominant during neocortical arousal, as reflected in electroencephalographic (EEG) low-voltage fast activity (LVFA) (Sherman, 1996), whereas burst firing is predominant during periods of drowsiness and slow wave sleep, as reflected in high-amplitude slow (i.e., delta) wave activity (Hirsch et al., 1983; McCormick, 1992; Sherman, 1996; Sherman and Guillery, 2001). Major contributors to the shift from burst to tonic firing include acetylcholine (ACh) from the peribrachial Ch-5 neuronal group (Sillito and Kemp, 1983; Eysel et al., 1986; Hu et al., 1989), norepinephrine from the locus ceruleus (DeLima and Singer, 1987), and histamine from the hypothalamic tuberomammillary nucleus (McCormick and Williamson, 1991).

Given that acoustic fear-arousing stimuli can affect the efficacy of stimulus processing in the dLGN, the question arises concerning the forebrain structures that contribute to this effect. The

Received June 11, 2002; revised Oct. 3, 2002; accepted Oct. 4, 2002.

This work was supported by National Science Foundation Grant IBN 9319699. We thank Daniel Ruttiman and Sanjeev Yadav for their technical assistance.

Correspondence should be addressed to Dr. Mary Eileen Cain, University of Kentucky, Department of Psychology, 109A Kastle Hall, Lexington, KY 40506-0044. E-mail: mecain2@uky.edu.

Copyright (C) 2002 Society for Neuroscience $0270-6474 / 02 / 2211026-09 \$ 15.00 / 0$ central nucleus of the amygdala (ACe) is a prime candidate based on a variety of observations. The ACe sends extensive projections to the brainstem, particularly to the region of cholinergic Ch-5 neurons (Hopkins and Holstege, 1978; Price and Amaral, 1981), the locus ceruleus (Price and Amaral, 1981; Van Bockstaele et al., 1998), and the tuberomammillary nucleus (Price and Amaral, 1981; Erickson et al., 1991). Neurons of the ACe are responsive to acoustic, fear-arousing, conditioned stimuli (Pascoe and Kapp, 1985; McEchron et al., 1995). The ACe has been shown to modulate the activity of brainstem cholinergic systems (Deboer et al., 1999), to contribute to the expression of a variety of responses during Pavlovian fear conditioning (Kapp et al., 1990; Davis, 1992; LeDoux, 1996), and to elicit arousal-related responses with stimulation (Ursin and Kaada, 1960; Applegate et al., 1983).

Based on these observations, it is hypothesized that the ACe contributes to conditioned thalamic arousal. Two experiments were performed to address this hypothesis. In experiment 1 , recordings from single dLGN neurons were taken during electrical stimulation of the ACe. It was predicted that stimulation would produce a shift from the burst firing mode to the tonic firing mode and an increase in the firing rate of dLGN neurons. In experiment 2, the region of the $\mathrm{ACe}$ was inactivated with muscimol, a $\mathrm{GABA}_{\mathrm{A}}$ agonist. It was predicted that inactivation would abolish the conditioned response characteristics of dLGN neurons to acoustic, fear-arousing, conditioned stimuli.

\section{MATERIALS AND METHODS}

Animals. Experimentally naive female New Zealand rabbits (Oryctolagus cuniculus) weighing $2.2-2.5 \mathrm{~kg}$ at the start of the experiment were used. They were maintained on a $12 \mathrm{hr}$ light/dark cycle and given food and water ad libitum. Principles for the care and use of laboratory animals in research were strictly followed, as outlined by the National Institutes of Health (1985). All procedures were approved by the University of Vermont Animal Care and Use Committee.

Surgery. After the application of a topical lidocaine solution (4\%) to 
the marginal ear vein, each rabbit was sedated with PromAce (acepromazine maleate, $4 \mathrm{mg} / \mathrm{kg}$, i.v.; Ayerst Laboratories, Rouses Point, NY) and anesthetized with sodium pentobarbital (30-60 mg/ $\mathrm{kg}$, i.v.). Each rabbit was placed in a Kopf stereotaxic instrument (David Kopf Instruments, Tujunga, CA) fitted with a rabbit headholder (model 1240; David Kopf Instruments). Using aseptic surgical procedures, three stainless steel jeweler's screws (Small Parts, Miami, FL) soldered to Amphenol male connectors (Wire Pro part 220-P02-100; Allied Electronics, Fort Worth, TX) for the attachment of wire leads were implanted to rest on dura and served as EEG electrodes. Two were placed bilaterally over the frontal cortex $(6.0 \mathrm{~mm}$ anterior and $3.0 \mathrm{~mm}$ lateral to bregma; bregma adjusted $1.5 \mathrm{~mm}$ above lambda), while a third was placed over the left parietal cortex $(13.0 \mathrm{~mm}$ posterior and $3.0 \mathrm{~mm}$ lateral to bregma). A fourth, similarly constructed electrode was implanted into the skull over the cerebellum to serve as a ground. A stainless steel well, designed to accommodate eccentric starr guides, and a miniature slave cylinder (Trent Wells, Coulterville, CA) was positioned over a 3.0-mm-diameter hole in the skull (6.5 $\mathrm{mm}$ posterior and $6.0 \mathrm{~mm}$ lateral to bregma) (Urban and Richard, 1792) to provide access to the dLGN region for neuronal recording. The well was secured in place with cyanoacrylate cement, swabbed with antibiotic (bacitracin), and sealed with a nylon plug. An indifferent electrode consisting of a $0.01-\mathrm{cm}$-diameter stainless steel wire was insulated with Formvar, except at the cross-sectional tip, and soldered to an Amphenol male connector. It was placed into the neocortex at a depth of $1.0 \mathrm{~mm}$. Rabbits were surgically prepared for either electrical stimulation of the ACe (experiment 1) or inactivation of the ACe with muscimol (experiment 2). To stimulate the ACe, an array of three stimulating electrodes, which permitted monopolar or bipolar stimulation, was positioned in the ACe $(0.5 \mathrm{~mm}$ anterior and $5.4 \mathrm{~mm}$ lateral to bregma; 11.9-12.0 ventral to dura) (Urban and Richard, 1792) ipsilateral to the dLGN recording site and secured in place with cyanoacrylate cement. The array allowed for multiple stimulation sites within the region of the $\mathrm{ACe}$ to correct for stereotaxic implantation error. The stimulating electrodes were constructed from stainless steel wire insulated with Formvar except for $\sim 250-500 \mu \mathrm{m}$ at the tip. Each was soldered to an Amphenol male connector and placed in a 19 gauge guide tube such that the bared tip of each of the three wires extended $\sim 4 \mathrm{~mm}$ beyond the end of the tube. The bared tips were separated by $\sim 0.5-0.75$ $\mathrm{mm}$. To inactivate the $\mathrm{ACe}$, a guide cannula, constructed from 22 gauge stainless steel hypodermic tubing and fitted with a 24 gauge stylet, was aimed $0.5 \mathrm{~mm}$ dorsal to the ACe $(0.5 \mathrm{~mm}$ anterior and $5.4 \mathrm{~mm}$ lateral to bregma; 11.4-11.5 mm ventral to dura) (Urban and Richard, 1792). It was located ipsilateral to the dLGN recording site and secured in place with cyanoacrylate cement to permit infusion of muscimol or PBS. Nylon bolts for head immobilization were attached to the skull with cyanoacrylate cement. The exposed skull was covered, and the wound was sealed with dental cement. Buprinex (buprenophine) was administered (0.005 $\mathrm{mg} / \mathrm{kg}$, s.c.), and each rabbit was returned to its home cage. Additional doses of Buprinex $(0.01 \mathrm{mg} / \mathrm{kg}$, s.c.) were administered postsurgically at $12 \mathrm{hr}$ intervals for $36 \mathrm{hr}$. A $7 \mathrm{~d}$ postoperative period ensued before the experiments began.

Apparatus. During the experiment, rabbits were placed in a Plexiglas rabbit restrainer with an adjustable headstock and backplate. The restrainer was placed within a shielded, ventilated, sound-attenuating chamber (Industrial Acoustics, Lodi, NJ), the inside of which was devoid of any internal or external illumination. This chamber was equipped with a $10.5 \times 19 \mathrm{~cm}$ speaker situated $24 \mathrm{~cm}$ in front of the rabbit's head and contained a modified stereotaxic frame that was used to immobilize the rabbit's head via attachment to the nylon head-immobilization bolts. Responses of single neurons were amplified with a high-impedance, differential preamplifier (06de3 DAM-6A; World Precision Instruments, Sarasota, FL), coupled with a main amplifier (06de3 75P11; Grass Instruments, Quincy, MA), for a total system gain of $\sim 25 \mathrm{k}$ and a bandwidth of $0.1-10 \mathrm{kHz}$. Responses from single neurons were fed into an audio monitor, a window discriminator (Frederick Haer \& Co., Bowdoinham, ME), and a computer (ABLE 40; New England Digital, Middletown, MA) for on-line data analysis. The computer also programmed the delivery of ACe stimulation trials and conditioning trials. Neuronal responses were displayed on a storage oscilloscope (model 5103N; Tektonix, Wilsonville, OR) and polygraph (model 78; Grass Instruments) and recorded on frequency modulation tape (model 4201, AR Vetter, Rebersberg, PA) along with verbal commentary, event markers, EEG, and heart rate. Acoustic CSs were generated with two separate voltagecontrolled generators (Wavetek model 111). Subsequent off-line analyses were performed using Biopac Student Lab Pro software (version 3.6;
Biopac Systems, Santa Barbara, CA), Biopac Acqknowledge software (version 3.5.3; Biopac Systems), and Data Acquisition and fast Fourier Analysis software programs (Med Associates, St. Albans, VT). Two independent output channels of a Grass S88 stimulator were used to administer the stimulation pulse trains $(500 \mathrm{msec}, 100 \mathrm{~Hz}, 0.5 \mathrm{msec}$ pulse duration), to administer the unconditioned stimulus (US) to the pinna during behavioral conditioning, and to produce direct current (DC) marker lesions at stimulation and recording sites.

Habituation to restraint. After surgical recovery, each rabbit in both experiments was habituated to the Plexiglas rabbit restrainer for $30 \mathrm{~min}$ a day for $6 \mathrm{~d}$. The modified stereotaxic frame was attached to the nylon head-immobilization bolts on each rabbit's head during the fifth and sixth days for the purpose of habituating each rabbit to head immobilization.

Experiment 1: stimulation electrode screening procedure. On the day after the last habituation session, each rabbit assigned to experiment 1 was administered an electrode screening procedure during which the stimulation electrodes were screened to determine whether they were placed accurately within the ACe. Different electrodes were tested each day, using either monopolar or bipolar stimulation $(100 \mathrm{~Hz}, 0.5 \mathrm{msec}$ pulse duration, $500 \mathrm{msec}$ train duration) in $25 \mu \mathrm{A}$ increments up to 250 $\mu \mathrm{A}$, and the rabbit was monitored for EEG and heart rate responses typically elicited by ACe stimulation in the rabbit (Applegate et al., 1983; Kapp et al., 1994). The presence of stimulation-induced EEG LVFA and a peak bradycardic response of $\geq 10 \%$ compared with the prestimulus period was assumed to indicate correct stimulation electrode placement within the ACe. The electrode configuration that elicited the most prominent responses with the least amount of applied current was selected for use in attempting to excite dLGN neurons. Rabbits that did not demonstrate the characteristic LVFA and bradycardic responses to stimulation during the electrode screening procedure were dropped from the experiment.

Experiment 2: behavioral training. On the day after the last habituation session as described above, a Pavlovian differential fear conditioning procedure was administered to each rabbit assigned to experiment 2 . Changes in heart rate and EEG were measured to assess differential conditioning to two acoustic CSs. The orienting phase of the Pavlovian conditioning procedure consisted of 18 pseudorandom presentations each of two tones of differing frequency $(500$ and $1300 \mathrm{~Hz}, 80 \mathrm{~dB}$, and 5.0 $\mathrm{sec})$. They were administered so that no more than two consecutive presentations of either tone were administered. These trials were necessary to habituate the cardiovascular and EEG orienting responses in the rabbit, which are characterized by bradycardia and a decrease in delta wave $(1-4 \mathrm{~Hz})$ activity with a simultaneous increase in LVFA in response to the presentation of novel stimuli. The conditioning phase began immediately after the orienting trials. Twenty-four pseudorandom presentations of each tone were administered, with the offset of one tone $(\mathrm{CS}+)$ being coincident with the onset of the US (500 msec, $1.0 \mathrm{~mA}, 60$ $\mathrm{Hz}$ stimulus train applied to the pinna). The US never followed the second tone (CS-). The US was administered via two stainless steel wire loops that pierced the pinna and were spaced $\sim 2.0 \mathrm{~cm}$ apart. They were inserted under local lidocaine anesthetic $24 \mathrm{hr}$ before behavioral conditioning. The designated tone frequencies for the CS + and $\mathrm{CS}-$ were counterbalanced among rabbits. To optimize conditioned discrimination between the $\mathrm{CS}+$ and the $\mathrm{CS}-$, a second differential conditioning session, again consisting of 24 presentations of each CS, was administered $24 \mathrm{hr}$ later.

EEG recording. Wire leads for EEG recording were attached to the male Amphenol connectors soldered to the stainless steel screws implanted to rest on dura. The EEG was filtered (filters set at $1-100 \mathrm{~Hz}$ ), amplified with a Grass model 7P511 amplifier, and displayed on-line using the Grass model 78 polygraph. The EEG was analyzed off-line by replaying the taped EEG into an analog-to-digital converter (Med Associates). It was then digitized using a sampling frequency of $128 \mathrm{~Hz}$ and subjected to Fourier analysis using the Data Acquisition and fast Fourier Analysis software package (Med Associates).

Heart rate recording. Electrodes for heart rate recording were placed by threading a stainless steel wire subcutaneously using a sterile, 21 gauge hypodermic needle. The electrodes were immediately removed after the experimental session. Heart rate was recorded using the Grass model 78 polygraph equipped with a cardiotachometer and was analyzed off-line using the Biopac Student Lab Pro software.

Electrophysiological recording. Electrophysiological recording sessions commenced in the sound-attenuating chamber $1 \mathrm{~d}$ after the completion of either the stimulation electrode screening procedure (experiment 1 ) or Pavlovian conditioning (experiment 2). Extracellular recordings from 


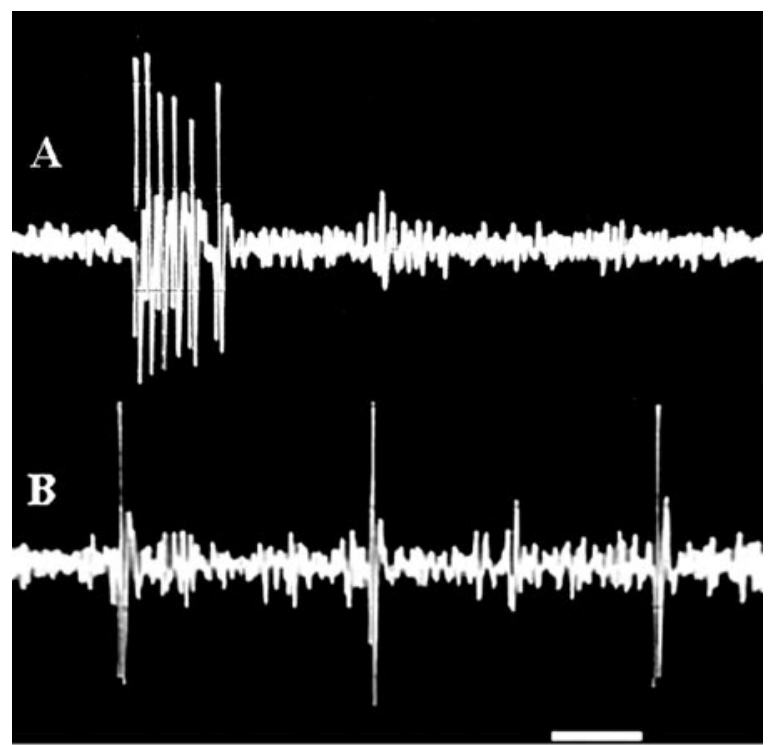

Figure 1. Oscilloscope traces depicting the burst firing $(A)$ and tonic firing $(B)$ modes of a representative thalamocortical dLGN neuron. Burst activity is typically observed during delta wave activity and tonic firing during LVFA. Calibration, $10 \mathrm{msec}$.

single neurons were made via platinized, tungsten microelectrodes (10 $\mathrm{M} \Omega$ tip impedance; Frederick Haer). The microelectrode was advanced to the dLGN through a 22 gauge guide tube by the use of a hydraulic microdrive (Trent Wells) attached to the miniature slave cylinder. Successive electrode penetrations occurred on subsequent days and were spaced from 0.2 to $2.0 \mathrm{~mm}$ apart by adjusting the position of the starr guide or by using starr guides of different eccentricity. Occasional, novel, acoustic stimuli were presented during neuronal recordings to aid in identifying dLGN neurons, because their activity is altered by novel acoustic stimuli (Cain et al., 1997). These acoustic stimuli consisted of white noise bursts and sounds produced from striking the external wall of the sound-attenuating chamber. The criteria for successful identification of a thalamocortical dLGN neuron was as follows: (1) a tonic firing mode characterized by a steady stream of action potentials, (2) a burst firing mode characterized by clusters of $2-10$ action potentials with an interspike interval (ISI) of $\leq 4 \mathrm{msec}$ and with silent periods of $\geq 50-100 \mathrm{msec}$ between bursts (Sherman, 1996), and (3) a switch from burst to tonic firing in response to novel acoustic stimuli (Cain et al., 2000). These criteria were used to distinguish thalamocortical neurons from interneurons. The latter rarely display tonic firing (Zhu et al., 1999). In addition, ACh inhibits dLGN interneurons (McCormick and Pape, 1988), and putative cholinergic neurons are activated by novel acoustic stimuli (Whalen et al., 1994; Silvestri and Kapp, 1998). Neurons that were inhibited in response to acoustic stimuli or that did not show a clear shift from the burst to tonic firing mode in response to these stimuli were not examined in this study. Although the inclusion of recordings from interneurons cannot be entirely ruled out in the present study, the above observations and the fact that the majority of the neurons in the dLGN are thalamocortical neurons ensures that the majority, if not all, of the recordings were from thalamocortical neurons. Figure 1 depicts the burst and tonic firing modes in a representative neuron. The criteria for successful isolation of a thalamocortical dLGN neuron were as follows: (1) waveform consistency during the isolation period, (2) a sufficient waveform amplitude to enable reliable and unambiguous discrimination, and (3) a 3:1 signal-to-noise ratio. Visual observations of the relationship between neuronal firing frequency and pattern and the state of the EEG were made on-line by inspection of the polygraph record.

Experiment 1: ACe stimulation. After isolation of a dLGN neuron, the ACe was stimulated using the stimulation electrodes that elicited the most characteristic responses during the electrode screening procedure $(500 \mathrm{msec}, 100 \mathrm{~Hz}, 0.5 \mathrm{msec}$ pulse duration at the intensity determined during the electrode screening procedure). Four to six stimulation trials were presented, with a variable 3-4 min intertrial interval. Marker lesions ( $100 \mu \mathrm{A}$ anodal DC for $5.0 \mathrm{sec})$ were made at designated recording sites at the end of selected, daily recording sessions. Marker lesions
$(200 \mu \mathrm{A}$ anodal $\mathrm{DC}$ for $5.0 \mathrm{sec})$ to determine the location of the stimulation electrode tips were made at least $24 \mathrm{hr}$ before the animals were killed.

Experiment 2: ACe inactivation. At least $24 \mathrm{hr}$ after the completion of training, the stylet was removed from the cannula, an injection stylet connected by polyethylene tubing to a $2.0 \mu \mathrm{l}$ Hamilton syringe was inserted partially into the cannula, and electrophysiological recording commenced in the sound-attenuating chamber. After isolation of a dLGN neuron, the injection stylet was lowered to a position $0.5 \mathrm{~mm}$ ventral to the cannula tip. The rabbit received either a $1.0 \mu$ l unilateral injection of muscimol $(0.5 \mu \mathrm{g}$ in sterile $0.9 \%$ PBS, $\mathrm{pH}$ 7.4) or vehicle (sterile PBS). Injections were made over a 2 min period using a Sage infusion pump. Previous research has demonstrated that an intracranial infusion of muscimol can affect behavior within 5 min (Manning, 1998; Spanis et al., 1999) and lasts $\sim 6 \mathrm{hr}$ ( $\mathrm{Li}$ et al., 1999). Therefore, a minimum of four presentations each of the CS + and CS - were administered in a pseudorandom sequence 5 min after the injection using a variable, $120 \mathrm{sec}$ intertrial interval. Each trial consisted of a $5 \mathrm{sec}$ pre-CS baseline period followed by a $5 \mathrm{sec}$ CS period. Marker lesions $(100 \mu \mathrm{A}$ anodal DC for $5.0 \mathrm{sec}$ ) were made at designated recording sites at the end of selected, daily recording sessions. Twenty-four hours after the recording of an individual neuron, additional blocks of conditioning trials (six $\mathrm{CS}+$ and six $\mathrm{CS}-$ ) with the US were administered as needed to maintain the conditioned discrimination across recording sessions. The following day, the same recording procedure was followed, and muscimol or PBS was infused based on random selection. Recording and additional conditioning trials continued for several sessions in this manner.

Histology. Rabbits were administered an overdose of sodium pentobarbital (Somelthal; $150 \mathrm{mg} / \mathrm{kg}$, i.v.) and perfused with saline followed by a $10 \%$ formol-saline solution. The brain was removed, and $60 \mu \mathrm{m}$ frozen serial sections were taken and stained with thionin. The locations of recording sites were determined microscopically by comparing microdrive coordinates with the relative electrode track positions and marker lesions.

Statistics. ANOVAs, $t$ tests for related measures, and standard scores ( $Z$ scores) were the primary statistical measures. The criterion for significance for all comparisons was $p<0.05$.

\section{RESULTS}

\section{Experiment 1: ACe stimulation}

\section{Histology}

Ten rabbits yielded a bradycardic response of $>10 \%$ during the electrode screening procedure used to determine accurate electrode placement within the ACe. Of these 10 rabbits, four demonstrated characteristic dLGN neurons during subsequent recording sessions and sufficient numbers of stimulation trials per recorded neuron for meaningful data analyses. Histological analyses revealed that these four rabbits exhibited stimulation sites within the ACe (Figs. 2, 3), and that all recorded neurons were located within the dLGN. The data from these four rabbits were used in the following analyses.

\section{Firing rate analysis}

Extracellular recordings were made from 23 neurons that (1) met the electrophysiological criteria for thalamocortical dLGN relay neurons (Sherman, 1996), (2) were located within the dLGN based on histological verification, (3) were tested for responsiveness to at least four ACe stimulation trials, and (4) met the electrophysiological criteria for single neuronal recordings as described previously (Pascoe and Kapp, 1985; Whalen et al., 1994). The predominant effect of stimulation of the ACe was an increase in firing rate. The mean firing rates during a $5 \mathrm{sec}$ prestimulus period (mean, 40.59; SE, 3.97) and during a $5 \mathrm{sec}$ stimulus period (mean, 77.82; SE, 4.83) commencing with stimulation onset were calculated across stimulation trials for each neuron. These mean firing rates were pooled across neurons, and a $t$ test for related measures revealed a significant increase in firing during the stimulus period $\left(t_{(22)}=10.01 ; p<0.0001\right) . Z$ 


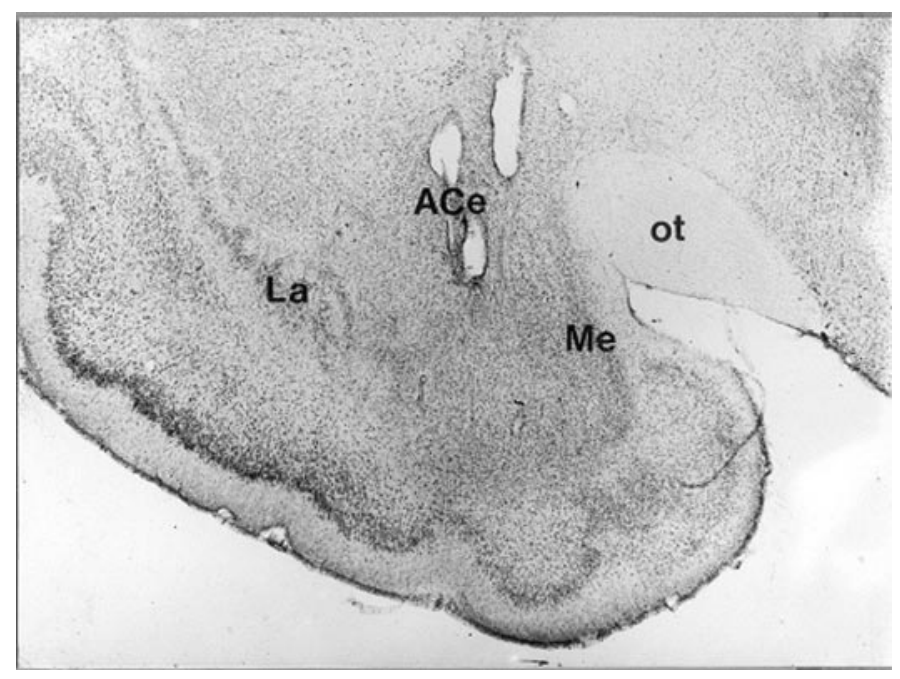

Figure 2. Photomicrograph of the stimulation electrode tip placements of a three-electrode stimulation array within the ACe of a representative rabbit. All electrode tips were located within the ACe. La, Lateral nucleus of the amygdala; $M e$, medial nucleus of the amygdala; ot, optic tract.

scores calculated from the spike counts during the $5 \mathrm{sec}$ prestimulus and $5 \mathrm{sec}$ stimulus periods of each stimulation trial revealed that 18 of these 23 neurons demonstrated statistically significant increases in activity after stimulation of the ACe $(Z=3.25-$ 38.48; $p<0.01)$. The remaining five neurons demonstrated a slight to moderate increase in firing rate, but the increase was not significant. An examination of the characteristics of these neurons did not distinguish them from those that demonstrated significant increases in firing rate.

An additional eight dLGN neurons were recorded during the presentation of three or fewer ACe stimulation trials. For each neuron, the mean firing rate across stimulation trials was calculated. These mean firing rates were pooled, and a $t$ test for related measures revealed a significant increase in firing in response to ACe stimulation $\left(t_{(7)}=3.78 ; p=0.007\right)$. The effect of ACe stimulation on the activity of a representative dLGN neuron is shown in Figure 4. Note in Figure $4 A$ that before stimulation, periods of delta wave activity predominated in the EEG, frequent neuronal bursts (represented by elongated tick marks) were apparent, and stimulation resulted in a shift to the tonic firing pattern (represented by short tick marks of higher frequency) that outlasted the duration of the stimulation, a commonly observed characteristic. However, as shown in Figure 4B, when LVFA prevailed immediately before stimulation, the neuron was in a tonic firing pattern and remained in that pattern during stimulation, which was commonly observed when stimulation was administered during LVFA and tonic firing.

\section{Onset latency analysis}

For each of the 31 neurons described above, $Z$ scores were calculated for each of the 10 consecutive $100 \mathrm{msec}$ bins during the first second of the stimulus periods by using the mean and SD of the last second of the prestimulus periods. The bin that contained the first significant $Z$ score was considered to represent the latency of the response of that neuron to stimulation. These analyses revealed that the median onset latency was 20-300 msec.

\section{Interspike interval analysis}

Interspike interval data were available for 25 of the 31 neurons described above. For these 25 neurons, a $t$ test for related mea-

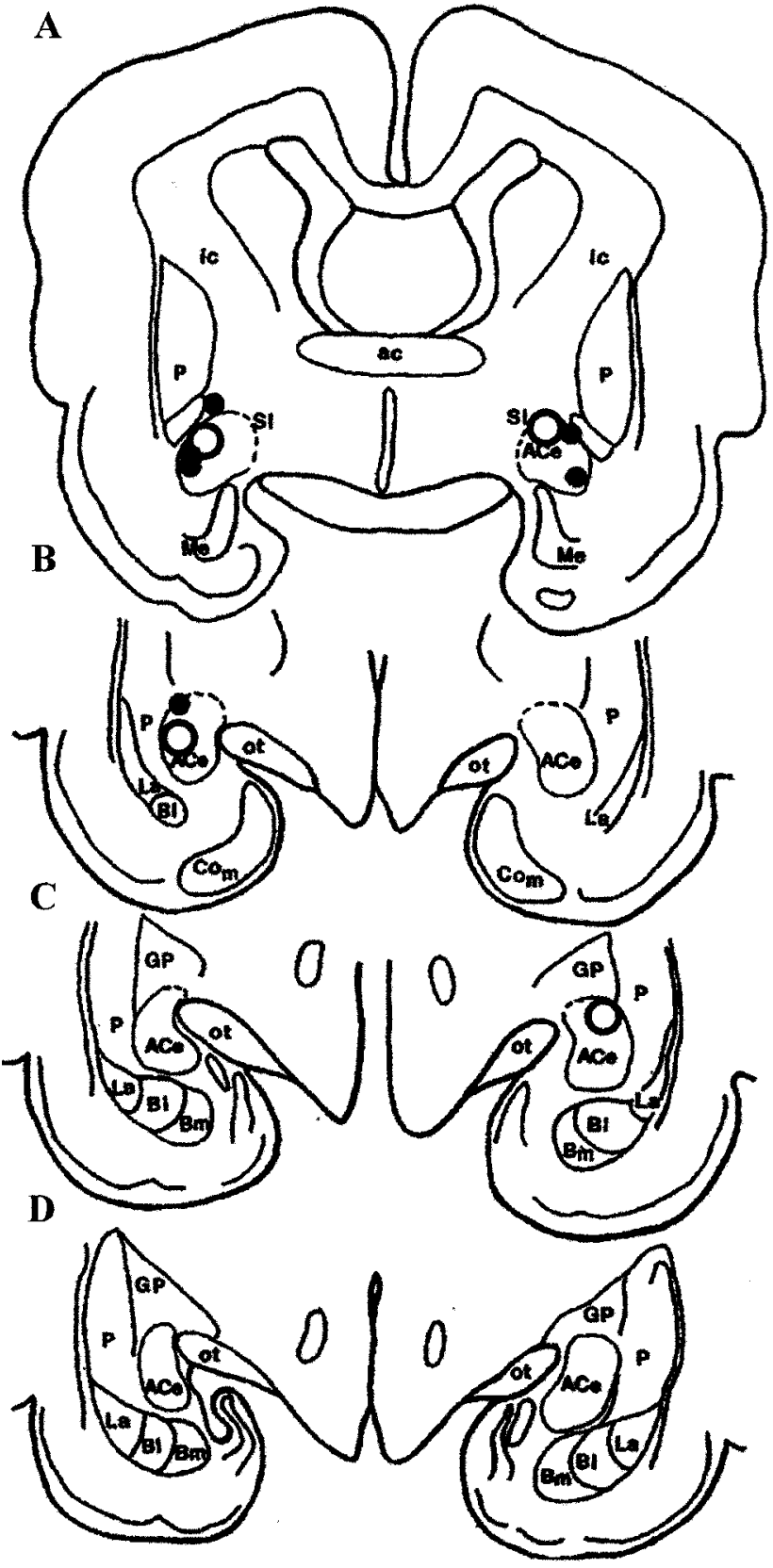

Figure 3. $A-D$, Line drawings of coronal sections depicting the distribution of placements of stimulation electrode arrays (open circles) and cannula tip placements (closed circles) within the amygdaloid central nucleus. Each open circle represents the approximate location of the tip of the stimulation electrode array for each rabbit included in this study. Each closed circle represents the location of the cannula tip for each rabbit included in this study. $A$ is most anterior; $D$ is most posterior. $a c$, Anterior commissure; $B l$, basolateral nucleus of the amygdala; $B m$, basomedial nucleus of the amygdala; Com, cortical nucleus of the amygdala; GP, globus pallidus; $i c$, internal capsule; $L a$, lateral nucleus of the amygdala; $M e$, medial nucleus of the amygdala; ot, optic tract; P, putamen; SI, sublenticular substantia innominata.

sures revealed a significantly greater percentage of ISIs $\left(t_{(24)}=\right.$ 2.24; $p=0.03$ ) that were $<4 \mathrm{msec}$ in duration during the $5 \mathrm{sec}$ prestimulus period (mean, 37.85; SE, 6.13) than during the $5 \mathrm{sec}$ stimulus period (mean, 22.35; SE, 4.78). As described in the EEG analysis section below, ACe stimulation significantly reduced the power of delta wave activity. These combined results demonstrate 
Figure 4. A, B, EEG, neuronal spikes of representative dLGN neurons, and cardiotachograph records recorded during representative ACe stimulation trials. Tick marks represent neuronal spikes. Elongated tick marks represent neuronal bursts. The arrowhead depicts the onset of the $500 \mathrm{msec}$ stimulation period. Bipolar stimulation current intensity was 250 $\mu \mathrm{A}$. Calibration, $1.0 \mathrm{sec}$. $A$, Polygraph trace during an ACe stimulation trial when EEG delta wave activity prevailed before stimulation. Note that the neuron is bursting during periods of EEG delta wave activity. Stimulation of the ACe produced LVFA, a shift from the burst to the tonic firing mode, and bradycardia. $B$, Polygraph trace during an ACe stimulation trial when EEG LVFA prevailed before stimulation. During stimulation of the $\mathrm{ACe}$, the EEG remained in LVFA, the neuron remained in tonic firing mode, and bradycardia was produced.
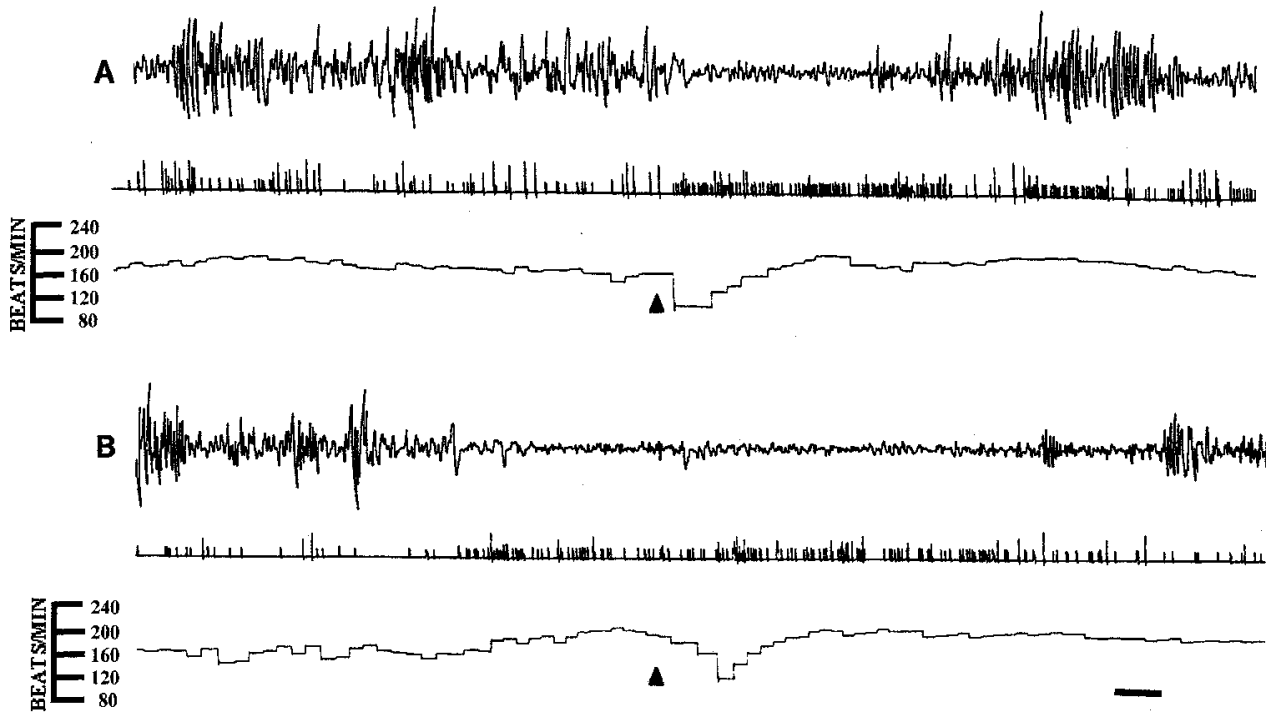

that greater delta wave activity and neuronal burst activity occurred during the prestimulus period than during the stimulus period, suggesting that stimulation of the ACe resulted in a decrease in the power of delta and an increase in tonic firing in dLGN neurons.

\section{Heart rate analysis}

Heart rate data were available during recordings from 14 of the 23 neurons for which recordings were taken during four or more stimulation trials. Heart rate data during recordings from the remaining nine neurons were not available because of equipment malfunctions. The duration of each $\mathrm{R}-\mathrm{R}$ wave interval for the five intervals that immediately preceded stimulation onset and for each interval for the five intervals that commenced after stimulation onset were determined and used to calculate heart rate during the prestimulus and stimulus periods. A $t$ test for related measures comparing the mean heart rate during the prestimulus periods (mean, 201.26; SE, 2.72) with the stimulus periods (mean, 180.93; SE, 3.38) pooled across stimulation trials for all neurons revealed a significant reduction in heart rate after stimulation $\left(t_{(13)}=5.09 ; p<0.0001\right) . t$ tests for related measures were performed on the data from each of the 14 neurons to determine whether the beats per minute during the stimulus period were significantly less than the beats per minute during the prestimulus period. The results demonstrated that significantly fewer beats per minute occurred during the stimulus period than during the prestimulus period during the recording of 11 of these 14 neurons $(p<0.05)$.

\section{EEG analysis}

EEG data during stimulation trials were available for 19 of the 23 neurons from which recordings were made during at least four stimulation trials. The data from the remaining four neurons were not available for analysis because of equipment malfunction. Fast Fourier analyses of EEGs yielded a numerical value representing the absolute power of delta (1-4 Hz) wave activity during each second of the $5 \mathrm{sec}$ prestimulus and stimulus periods commencing with stimulation onset. A $t$ test for related measures comparing the power of delta wave activity during the first $4 \mathrm{sec}$ of the prestimulus period (mean, 16.79; SE, 0.61) and the last $4 \mathrm{sec}$ of the stimulus period (mean, 6.85; SE, 0.45) pooled across stimu- lation trials for all neurons showed a significant reduction in delta wave activity in response to stimulation $\left(t_{(18)}=6.12 ; p<0.0001\right)$. (The EEG data for the $1 \mathrm{sec}$ periods immediately before and after stimulation onset were not used in the analysis to mitigate the possibility of any contamination of the EEG by stimulation artifact.) $t$ tests for related measures were performed on the data from each of these 19 neurons to determine whether the power of delta during the stimulus period was significantly less than it was during the prestimulus period. The results demonstrated that the power of delta during the stimulus period was significantly less than during the prestimulus period for 15 of these 19 neurons $(p<0.05)$.

\section{Experiment 2: ACe inactivation Histology}

Only data from those rabbits that (1) had histologically verified recording electrode placements in the dLGN, (2) had a histologically verified cannula placement within $0.5 \mathrm{~mm}$ of the ACe (Fig. 3), (3) acquired differential Pavlovian heart rate conditioning, and (4) yielded characteristic dLGN neurons during recording sessions were analyzed. Six rabbits met the above criteria, and their data were used in the following analyses. As depicted in Figure 3, cannula placements were located in the more anterior region of the Ace, where it extends into the sublenticular substantia innominata (SSI).

\section{Heart rate and EEG conditioned responding}

Percentage changes in heart rate during each trial during the orienting and conditioning phases were calculated by subtracting the number of beats during the $5 \mathrm{sec} C \mathrm{CS}$ period from the number of beats during the $5 \mathrm{sec}$ pre-CS baseline period and dividing that value by the number of beats during the pre-CS period. A twofactor $(\mathrm{CS} \times$ trials) within-subjects ANOVA revealed no significant effects of $\mathrm{CS}$, trials, or a CS $\times$ trials interaction for heart rate percentage changes during the orienting phase $(F<1)$. However, during the conditioning phase, these six rabbits demonstrated differential heart rate responses to the CS+ and CS-, with greater bradycardia elicited during the CS+. A two-factor (CS type $\times$ day) within-subjects ANOVA performed on heart rate data of the conditioning phase revealed a significant effect of CS type $\left(F_{(1,116)}=72.87 ; p<0.0001\right)$, a significant effect of day 


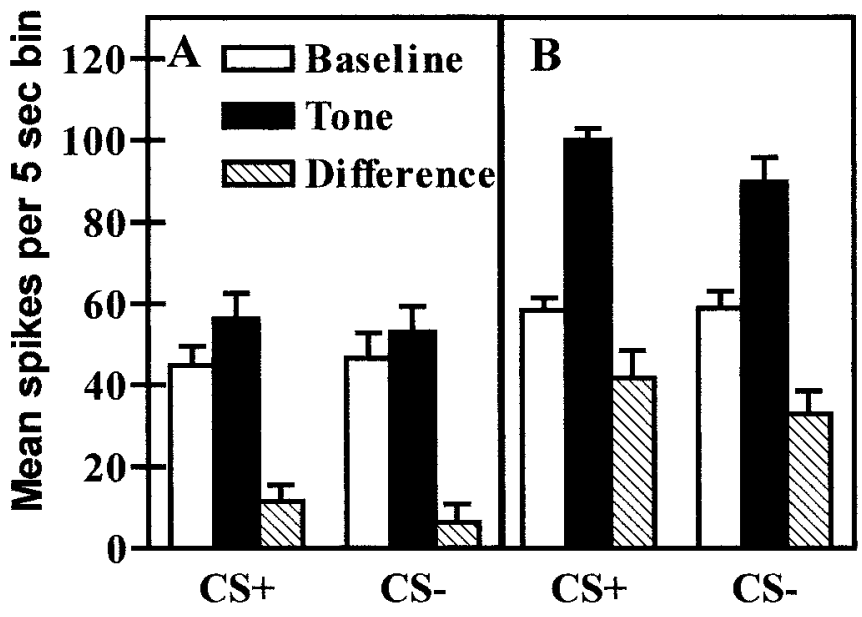

Figure 5. $A, B$, Mean \pm SEM activity of 33 neurons recorded from the dLGN during either muscimol $(A)$ or PBS $(B)$ infusion into the region of the ACe. The mean numbers of spikes during the $5 \mathrm{sec}$ pre-CS baseline period, the $5 \mathrm{sec}$ CS period, and the difference scores are shown for both $\mathrm{CS}+$ and $\mathrm{CS}-$ presentations.

$\left(F_{(1,116)}=49.00 ; p<0.0001\right)$, and a significant interaction between CS type and day $\left(F_{(1,116)}=21.33 ; p<0.0001\right)$. These results demonstrate the acquisition of discriminative heart rate responding during the conditioning phase, with significantly greater bradycardia in response to the $\mathrm{CS}+$.

Fast Fourier analysis of EEGs yielded a numerical value representing the absolute power of delta wave activity $(1-4 \mathrm{~Hz})$ during each second of the $5 \mathrm{sec}$ pre-CS and $5 \mathrm{sec} C S$ periods. The first $4 \mathrm{sec}$ of the pre-CS baseline and the last $4 \mathrm{sec}$ of the CS presentation were averaged for each pre-CS baseline and CS presentation. The percentage change in delta to each CS was determined by subtracting the power of delta during the last $4 \mathrm{sec}$ of the CS period from the first $4 \mathrm{sec}$ of the pre-CS period and dividing this value by the power of delta during the pre-CS period. A two-factor $(\mathrm{CS} \times$ trials) within-subjects ANOVA revealed no significant effect of $\mathrm{CS}$, trials, or a $\mathrm{CS} \times$ trials interaction for delta wave percentage changes during the orienting phase. However, during the conditioning phase these six rabbits demonstrated differential EEG delta wave activity in response to the $\mathrm{CS}+$ and $\mathrm{CS}-$, with a greater decrease in delta wave activity during the $\mathrm{CS}+$. A two-factor (CS type $\times$ day) within-subjects ANOVA performed on delta wave data of the conditioning phase revealed a significant effect of CS type $\left(F_{(1,116)}=7.26 ; p=0.008\right)$ and day $\left(F_{(1,116)}=8.34 ; p=0.005\right)$ but no significant interaction between CS type and day $\left(F_{(1,116)}=1.34 ; p>0.05\right)$. These results demonstrate discriminative EEG responding during the conditioning phase, with significantly greater decreases in delta in response to the $\mathrm{CS}+$.

\section{Neuronal analysis}

Recordings were made from 33 neurons, each of which was recorded during at least four $\mathrm{CS}+$ and four $\mathrm{CS}-$ presentations. Nineteen neurons were recorded during PBS administration, and 14 neurons were recorded during muscimol administration. As is apparent from Figure 5, the pooled data from these 33 neurons across all CS trials and drug conditions of the recording sessions demonstrated that muscimol administration markedly attenuated the response of dLGN neurons to CS presentations compared with PBS administration. This effect was confirmed using a $2 \times$ $2 \times 2$ (drug $\times$ time $\times$ CS type $)$ mixed factorial ANOVA with drug (muscimol vs PBS) as a between-subjects factor and time (baseline vs tone) and CS type (CS+ vs $\mathrm{CS}-$ ) as within-subjects factors. The analysis revealed a main effect of drug $\left(F_{(1,31)}=\right.$ $18.592 ; p<0.001)$ and time $\left(F_{(1,31)}=33.706 ; p<0.001\right)$ but not CS type $\left(F_{(1,31)}=1.674 ; p=0.205\right)$. These main effects demonstrated the attenuating effect of muscimol on CS neuronal responses (drug effect), the responsiveness of dLGN neurons to the CSs (time effect), and the lack of discriminative neuronal responses to the two CSs (no CS type effect).

The interactions between time $\times \operatorname{drug}\left(F_{(1,31)}=12.286 ; p<\right.$ $0.001)$ and time $\times$ CS type were significant $\left(F_{(1,31)}=6.186 ; p=\right.$ 0.018), demonstrating a differential effect of drug administration on CS responsiveness (time $\times$ drug) and a differential neuronal response to $\mathrm{CS}+$ versus $\mathrm{CS}-$ presentations (time $\times \mathrm{CS}$ type). There were no significant interactions between CS type and drug $(F<1)$ and time, CS type, and drug $(F<1)$. To better understand the interactions, simple effects analyses incorporating a Bonferroni correction ( $p=0.01$ ) were performed. The results revealed several statistically significant findings. First, there was a significant increase in firing rate during CS + presentations compared with the pre-CS + baseline firing rate during both musci$\operatorname{mol}\left(F_{(1,31)}=11.35 ; p<0.01\right)$ and PBS $\left(F_{(1,31)}=200.65 ; p<\right.$ $0.01)$ administration. According to estimates by Cohen (1988), examination of the effect size $(d)$ suggested that there was a moderate increase in firing to $\mathrm{CS}+$ presentations during muscimol administration $(d=0.57)$, whereas there was a large increase in firing during PBS administration $(d=1.98)$. This result indicates that muscimol attenuated increases in firing during CS+ presentations. Second, there was a significant increase in firing rate during $\mathrm{CS}-$ presentations compared with the pre-CS - baseline firing rate during PBS administration $\left(F_{(1,31)}=110.55 ; p<\right.$ $0.01)$ but not during muscimol administration $\left(F_{(1,31)}=3.48 ; p>\right.$ $0.01)$. This result demonstrated that muscimol decreased CSresponsiveness. Third, there was a significant increase in firing rate during $\mathrm{CS}+$ presentations compared with that of $\mathrm{CS}-$ presentations during PBS administration $\left(F_{(1,31)}=12.14 ; p<\right.$ $0.01)$ but not during muscimol administration $(F<1)$, demonstrating that muscimol attenuated the differential increase in firing during CS presentations. Finally, planned comparisons revealed that the pre-CS baseline firing rate, regardless of trial type, was significantly attenuated during muscimol administration compared with PBS administration $\left(t_{(64)}=3.08 ; p<0.01\right)$. This result, together with the effects of muscimol on neuronal responses to CS presentations described above, demonstrated that muscimol exerted a general attenuation of the activity of dLGN neurons.

\section{Heart rate}

The percentage changes in heart rate to both $\mathrm{CS}+$ presentations and $\mathrm{CS}-$ presentations were calculated during the recording of the 33 neurons described above. As is apparent from Figure $6 A$, the pooled percentage changes in heart rate suggest that muscimol administration did not attenuate the bradycardia response to $\mathrm{CS}+$ presentations. This lack of an effect was confirmed using a two-factor (CS type $\times$ drug) mixed factorial ANOVA with CS type as the within-subjects factor and drug as a between-subjects factor. The analysis revealed a significant effect of CS type $\left(F_{(1,213)}=130.50 ; p<0.0001\right)$ but no significant effect of drug $(F<1)$ and no significant interaction between CS type and drug $\left(F_{(1,213)}=1.27 ; p=0.26\right)$. These results demonstrate the presence of differential bradycardic responses to the CSs, and that muscimol did not disrupt this differential response. 


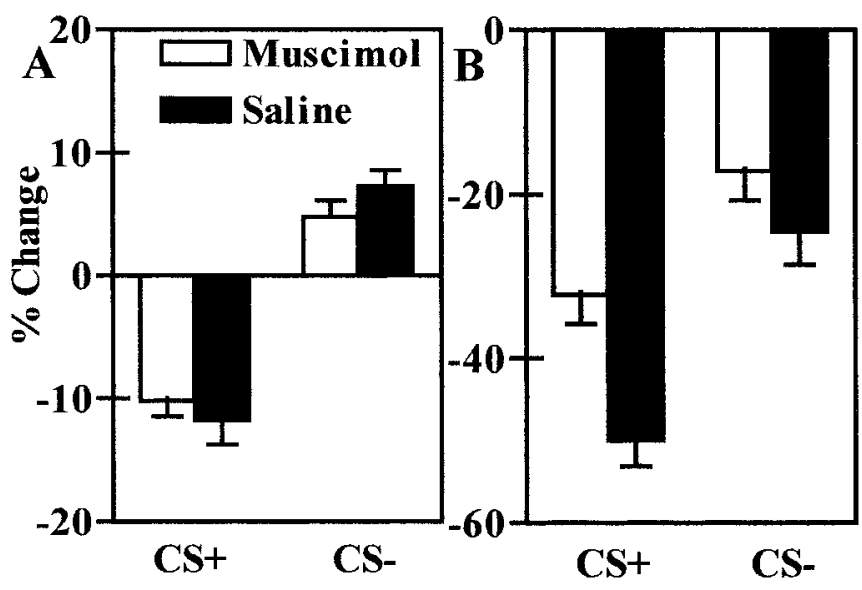

Figure 6. $A$, Mean \pm SEM percentage change in heart rate during CS presentations during the recording of the 33 neurons described above. $B$, Mean \pm SEM percentage change in the absolute power of delta $(1-4 \mathrm{~Hz})$ wave activity in the EEG during CS presentations during the recording of the 33 neurons described above.

\section{EEG}

The percentage changes in EEG delta wave activity to both CS+ and $\mathrm{CS}-$ presentations were calculated during the recording of the 33 neurons described above. As is apparent from Figure $6 B$, the pooled percentage changes in delta wave activity suggest that significantly greater percentage changes occurred in delta wave activity during $\mathrm{CS}+$ presentations than during $\mathrm{CS}-$ presentations, and that muscimol administration attenuated the responses to the CSs. This was confirmed with a two-factor (CS type $\times$ drug) mixed factorial ANOVA, with CS type as the withinsubjects factor and drug as a between-subjects factor. The analysis revealed a significant effect of CS type $\left(F_{(1,218)}=38.06 ; p<\right.$ $0.0001)$ and drug $\left(F_{(1,218)}=9.93 ; p=0.002\right)$ but no significant interaction between CS type and drug $\left(F_{(1,218)}=2.64 ; p=0.11\right)$. These results demonstrate the presence of differential EEG responding, and that muscimol attenuated CS responding.

\section{CS onset latency analysis}

The median onset latency for the 33 neurons described above to $\mathrm{CS}+$ presentations during PBS administration was from 201 to $300 \mathrm{msec}$, whereas that during muscimol administration was from 301 to 400 msec. A Mann-Whitney $U$ test revealed that the onset latencies for $\mathrm{CS}+$ trials during PBS administration did not differ from those during muscimol administration $(Z=0.79 ; p=0.43)$. The median onset latency to $\mathrm{CS}-$ presentations during both PBS and muscimol administration was from 201 to 300 msec. A MannWhitney $U$ test revealed that the onset latencies for CS - trials during PBS administration did not differ from those during muscimol administration $(Z=0.52 ; p=0.61)$. These combined results demonstrate that muscimol did not affect the onset latencies of dLGN neurons during both $\mathrm{CS}+$ and $\mathrm{CS}-$ trials.

\section{DISCUSSION}

Experiment 1 demonstrated that stimulation of the ACe increased the firing rate and decreased the percentage of interspike intervals that are indicative of burst activity in the majority of dLGN neurons, thereby inducing a tonic firing mode in these neurons. Experiment 2 demonstrated that muscimol infused into the anterior region of the amygdala, where the $\mathrm{ACe}$ and its extension into the SSI are located (i.e., the central extended amygdala), markedly attenuated the response of dLGN neurons to CS presentations. These combined results provide support for the hypothesis that the ACe contributes to an enhanced state of arousal during learned fear (Kapp and Cain, 2001).

\section{ACe stimulation}

The tonic firing mode induced in dLGN neurons by ACe stimulation is the most efficient mode for relaying visual information from the retina to the visual cortex (Sherman and Guillery, 2001). Tonic firing promotes linear transformation between the visual stimulus and the response (Sherman and Guillery, 2001) as opposed to the burst firing mode that imparts a nonlinear distortion in the relay of signals from the retina to the cortex (Sherman, 1996; Sherman and Guillery, 2001; Swadlow and Gusev, 2001).

In addition to affecting the firing rate and mode of dLGN neurons, stimulation of the ACe produced decreases in the power of delta wave activity in the EEG and short-latency bradycardic responses, which are characteristic of ACe electrical stimulation in the rabbit (Kapp et al., 1982, 1994; Cox et al., 1987). It has been demonstrated that those ACe sites that elicit bradycardia in the rabbit during electrical stimulation also elicit a number of other responses that are indicative of an enhanced state of arousal (Applegate et al., 1983). This constellation of elicited responses has led to the hypothesis that the ACe contributes to a heightened state of arousal in response to emotionally laden stimuli, that many of these responses enhance sensory information processing, and that the forebrain and brainstem projections of the ACe provide the substrate for their expression (Kapp et al., 1990, 1992; Kapp and Cain, 2001). The current finding that electrical stimulation of the ACe elicits a firing mode in dLGN neurons that renders them more sensitive to retinal input is consistent with this hypothesis.

\section{Muscimol inactivation of the $\mathrm{ACe}$}

The results of experiment 2 demonstrated that muscimol attenuated (1) increases in the firing of dLGN neurons to both CS types, (2) differential responses of these neurons to the two CSs, and (3) pre-CS baseline firing. Muscimol was chosen to inactivate the ACe because of the intense GABAergic innervation of the brainstem projections originating from the ACe (Nose et al., 1991; Smith and Pare, 1994; Cassell et al., 1999). Therefore, it is reasonable to assume that muscimol inhibited activity in brainstem projection neurons. Whereas injections ipsilateral to the dLGN recording site markedly attenuated the response of dLGN neurons to CS presentations, they did not affect the bradycardic response to CS presentations, most likely because of the use of unilateral injections.

With respect to the area within the amygdala affected by muscimol, a $1.0 \mu \mathrm{l}$ infusion volume was chosen to increase the probability that a substantial amount of tissue within the ACe would be affected. This volume most probably affected tissue in addition to the ACe. Therefore, it cannot be concluded with certainty that the observed effects on dLGN neuronal activity were attributable solely to inactivation of the ACe to the exclusion of inactivation of other amygdaloid or basal forebrain tissue. In this regard, two points are worth emphasizing. First, in the rabbit, the largest areal extent of the ACe $(2.3 \mathrm{~mm}$ medial to lateral, $2.0 \mathrm{~mm}$ rostral to caudal, $2.4 \mathrm{~mm}$ dorsal to ventral) is located anteriorly (Urban and Richard, 1792), and the cannula placements were located in this region, as is apparent from Figure 3. As is also apparent from Figure 3, this location is considerably anterior and dorsomedial to the largest areal extent of the basolateral and lateral amygdaloid nuclei, which could be considered 
as likely candidates for mediating the muscimol effect because of their contribution to fear conditioning (Muller et al., 1997; Wilensky et al., 1999). Although assigning the observed effects of muscimol to an effect on these nuclei cannot be ruled out, their location is somewhat distant from the anterior ACe injection sites, thereby minimizing its spread from the injection site into these nuclei. Second, with respect to the extra-amygdaloid tissue that may contribute to the observed effects of muscimol, the cannula placements in the present research were located in the anterior region of the $\mathrm{ACe}$, as described above. A potential extra-amygdaloid site of muscimol action in this region is the SSI. This area contains substantial numbers of neurons of the ACe, known as the central extended amygdala, as they course in a rostral-medial direction through the basal forebrain (De Olmos and Heimer, 1999). Although the possibility exists that muscimol exerted its effect on neurons other than those of the ACe in this region, it is also quite likely that $\mathrm{ACe}$ neurons in this region were affected by muscimol. In summary, it would appear that the most parsimonious explanation for the observed effects of muscimol is that it exerted its affects on dLGN neuronal activity by inhibiting the activity of ACe brainstem projection neurons.

Muscimol inactivation of the amygdala, and in particular the basolateral amygdaloid complex (BLA), blocks the development of neuronal plasticity in the thalamic medial geniculate nucleus (MGN) during instrumental avoidance (Poremba and Gabriel, 2001) and Pavlovian fear conditioning (Maren et al., 2001). These results and the present results illustrate the pervasive influence of the amygdala on thalamic activity during aversive conditioning. Although the precise pathways by which the BLA affects the MGN are unknown, the possibility exists that the projections from the BLA to the ACe comprise one important component. Additional research will be required to assess the extent to which the ACe also affects the development of MGN neuronal plasticity during aversive conditioning.

\section{Neural systems mediating conditioned thalamic arousal}

The effects of ACe stimulation and inactivation on dLGN neuronal firing are consistent with previous anatomical and electrophysiological findings. The ACe projects to the region of the cholinergic Ch-5 neuronal group (Hopkins and Holstege, 1978; Price and Amaral, 1981), the noradrenergic locus ceruleus (Van Bockstaele et al., 1998), and the histaminergic tuberomammillary nucleus (Price and Amaral, 1981; Erickson et al., 1991), all of which project to the dLGN (DeLima and Singer, 1987; Steriade and Llinas, 1988; McCormick and Williamson, 1991). Electrical stimulation of all of these areas, or application of their neurotransmitters onto dLGN neurons, shifts the firing pattern of dLGN neurons from burst to tonic (Kayama, 1985; Uhlrich et al., 1990, 2002). Additional research will be required to determine which if any of these systems mediate the influence of the ACe on dLGN neurons.

The projections from the ACe to the above regions suggest that activation of the ACe would affect the dLGN neurons with rather short onset latencies. For example, stimulation of the $\mathrm{Ch}-5$ region in the cat excites dLGN neurons with latencies of $<100 \mathrm{msec}(\mathrm{Hu}$ et al., 1989), and stimulation of the tuberomammillary nucleus in the cat produces an immediate increase in the spontaneous activity of dLGN neurons (Uhlrich et al., 2002). Stimulation of the $\mathrm{ACe}$ in rabbits excites neurons within the $\mathrm{Ch}-5$ cell region in $<10$ msec (Silvestri and Kapp, 1998). Hence, shorter onset latencies in response to $\mathrm{ACe}$ stimulation or to $\mathrm{CS}$ presentations than those observed (201-300 msec) were predicted, if indeed such stimulation were mediated via the Ch-5 cell group or the tuberomammillary nucleus. Several possible mechanisms may account for the long onset latencies observed. First, activation of the ACe in the awake rabbit through some undetermined mechanism may have recruited inhibition at an unknown junction in the circuit, thereby counterbalancing any immediate onset excitatory effects. Second, the possibility exists that glutamate-containing corticogeniculate axons, which originate in the visual cortex and terminate in the dLGN, may have mediated the effect of ACe stimulation on dLGN neurons. Although the ACe does not project directly to the visual cortex, it may influence the visual cortex by an undetermined circuitous route that may account for the long activation latencies.

Overall, the results of these experiments are consistent with the hypothesis that at least one function of the ACe is to increase arousal, which is manifested in a variety of responses that function to enhance sensory processing (Kapp et al., 1990, 1992; Kapp and Cain, 2001). These responses are mediated by the various projections of the ACe and represent a coordinated pattern of responses that can best be interpreted within an arousal framework. Given the role of the ACe in arousal and the expression of a variety of responses in the presence of fear-arousing stimuli (Davis, 1992; Kapp et al., 1992, LeDoux, 1996), the results of this research are consistent with the notion that the ACe contributes to the most accurate perception of sensory stimuli to enable an organism to respond appropriately when confronted with a fearful situation.

\section{REFERENCES}

Applegate CD, Kapp BS, Underwood MD, McNall CJ (1983) Autonomic and somatomotor effects of amygdala central nucleus stimulation in awake rabbits. Physiol Behav 31:353-360.

Cain ME, Kapp BS, Silvestri AJ, Guarraci FA (1997) The effects of arousal-enhancing acoustic stimuli on the activity of neurons within the dorsal lateral geniculate nucleus of the awake rabbit. Soc Neurosci Abstr 22:2049.

Cain ME, Kapp BS, Puryear CB (2000) Arousal-related associative response characteristics of dorsal lateral geniculate nucleus neurons during acoustic Pavlovian fear conditioning. Behav Neurosci 114:241-253.

Cassell MD, Freedman LJ, Shi C (1999) The intrinsic organization of the central extended amygdala. In: Advancing from the ventral striatum to the extended amygdala: implications for neuropsychiatry and drug abuse (McGinty JF, ed), pp 217-241. New York: The New York Academy of Sciences.

Cohen J (1988) Statistical power analysis for the behavioral sciences, Ed 2. New York: Academic.

Cox GE, Jordan D, Paton JF, Spyer KM, Wood LM (1987) Cardiovascular and phrenic nerve responses to stimulation of the amygdala central nucleus in the anaesthetized rabbit. J Physiol 389:541-556.

Davis M (1992) The role of the amygdala in conditioned fear. In: The amygdala: neurobiological aspects of emotion, memory, and mental dysfunction (Aggleton JP, ed), pp 255-306. New York: Wiley.

Davis M, Whalen PJ (2001) The amygdala: vigilance and emotion. Mol Psychiatry 6:13-34.

Deboer T, Ross RJ, Morrison AR, Sanford LD (1999) Electrical stimulation of the amygdala increases the amplitude of elicited pontogeniculo-occipital waves. Physiol Behav 66:119-124.

DeLima AD, Singer W (1987) The brainstem projection to the lateral geniculate nucleus in the cat: identification of cholinergic and monoaminergic elements. J Comp Neurol 259:92-191.

De Olmos JS, Heimer L (1999) The concepts of the ventral striatopallidal system and extended amygdala. In: Advancing from the ventral striatum to the extended amygdala: implications for neuropsychiatry and drug abuse (McGinty JF, ed), pp 1-32. New York: The New York Academy of Sciences.

Erickson H, Blomqvist A, Kohler C (1991) Origin of neuronal inputs to the region of the tuberomammillary nucleus in the rat brain. J Comp Neurol 311:45-64.

Eysel UT, Pape HC, Van Schayck R (1986) Excitatory and differential disinhibitory actions of acetylcholine in the lateral geniculate nucleus of the cat. J Physiol 370:233-254.

Hirsch JC, Fourment A, Marc ME (1983) Sleep-related variations of 
membrane potential in the lateral geniculate body relay neurons of the cat. Brain Res 259:308-312.

Hopkins DA, Holstege G (1978) Amygdaloid projections to the mesencephalon, pons, and medulla oblongata in the cat. Exp Brain Res 32:529-547.

Hu B, Steriade M, Deschenes M (1989) The effects of brainstem peribrachial stimulation on neurons of the lateral geniculate nucleus. Neuroscience 31:13-24.

Kapp BS, Cain ME (2001) The neural basis of arousal. In: International encyclopedia of the social and behavioral sciences (Smelser NJ, Battes PB, eds), pp 754-758. New York: Elsevier.

Kapp BS, Gallagher M, Underwood MD, McNall CL, Whitehorn D (1982) Cardiovascular responses elicited by electrical stimulation of the amygdala central nucleus in the rabbit. Brain Res 234:251-262.

Kapp BS, Wilson A, Pascoe JP, Supple W, Whalen PJ (1990) A neuroanatomical systems analysis of bradycardia in the rabbit. In: Neurocomputation and learning: foundations of adaptive networks (Gabriel M, Moore J, eds), pp 53-90. Cambridge, MA: Bradford and MIT.

Kapp BS, Whalen PJ, Supple W, Pascoe JP (1992) Amygdaloid contributions to conditioned arousal and sensory information processing. In: The amygdala: neurobiological aspects of emotion, memory, and mental dysfunction (Aggleton JP, ed), pp 229-254. New York: Wiley.

Kapp BS, Supple WF, Whalen PJ (1994) The effects of electrical stimulation of the amygdaloid central nucleus on neocortical arousal in the rabbit. Behav Neurosci 108:353-360.

Kayama Y (1985) Ascending, descending, and local control of neuronal activity in the rat lateral geniculate nucleus. Vision Res 25:339-347.

LeDoux J (1996) The emotional brain: the mysterious underpinnings of emotional life. New York: Simon and Schuster.

Li CR, Mazzoni P, Anderson RA (1999) Effects of reversible inactivation of macaque lateral intraparietal area on visual and memory saccades. J Neurophysiol 81:1827-1838.

Manning BH (1998) A lateralized deficit in morphine antinociception after unilateral inactivation of the central amygdala. $\mathrm{J}$ Neurosci 18:9453-9470.

Maren S, Yap S, Goosens K (2001) The amygdala is essential for the development of neuronal plasticity in the medial geniculate nucleus during auditory fear conditioning in rats. J Neurosci 21:RC135(1-6).

McCormick DA (1992) Neurotransmitter actions in the thalamus and cerebral cortex and their role in the neuromodulation of thalamocortical activity. Prog Neurobiol 39:337-388.

McCormick DA, Pape HC (1988) Acetylcholine inhibits identified interneurons in the cat lateral geniculate nucleus. Science 334:246-248.

McCormick DA, Williamson A (1991) Modulation of neuronal firing mode in the cat and guinea pig dorsal lateral geniculate nucleus by histamine: possible cellular mechanisms of histaminergic control of arousal. J Neurosci 11:3188-3199.

McEchron MD, McCabe PM, Green EJ, Llabre MM, Schneidermann NM (1995) Simultaneous single unit recordings in the medial geniculate nucleus and amygdaloid central nucleus through habituation, acquisition, and extinction of rabbits classically conditioned heart rate. Brain Res 682:157-166.

Muller J, Corodimas KP, Fridel Z, LeDoux JE (1997) Functional inactivation of the lateral and basal nuclei of the amygdala by muscimol infusion prevents fear conditioning to an explicit conditioned stimulus and to contextual stimuli. Behav Neurosci 111:683-691.
Nose I, Higashi H, Inokuchi H, Nishi S (1991) Synaptic responses of guinea pig and rat central amygdala neurons in vitro. J Neurophysiol 65:1227-1241.

Pascoe J, Kapp BS (1985) Electrophysiological characteristics of amygdaloid central nucleus neurons in the awake rabbit. Brain Res Bull 14:331-338.

Poremba A, Gabriel M (2001) Amygdalar efferents initiate auditory thalamic discriminative training-induced neuronal activity. J Neurosci 21:270-278.

Price JL, Amaral DG (1981) An autoradiographic study of the projections of the central nucleus of the monkey amygdala. J Neurosci 1:1242-1259.

Sherman SM (1996) Dual response modes in lateral geniculate neurons: mechanisms and functions. Vis Neurosci 13:205-213.

Sherman SM, Guillery RW (2001) Exploring the thalamus. San Diego: Academic.

Sillito AM, Kemp JA (1983) Cholinergic modulation of the functional organization of the cat visual cortex. Brain Res 289:143-155.

Silvestri AJ, Kapp BS (1998) Amygdaloid modulation of mesopontine peribrachial neuronal activity: implications for arousal. Behav Neurosci 112:571-588.

Smith Y, Pare D (1994) Intra-amygdaloid projections of the lateral nucleus in the cat: PHA-L anterograde labeling combined with postembedding GABA and glutamate immunocytochemistry. J Comp Neurol 342:232-248.

Spanis CW, Bianchin MM, Izquierdo I, McGaugh JL (1999) Excitotoxic basolateral amygdala lesions potentiate the memory impairment of muscimol injected into the medial septal area. Brain Res 816:329-336.

Steriade M, Llinas RR (1988) The functional states of the thalamus and the associated neuronal interplay. Physiol Rev 68:649-742.

Swadlow HA, Gusev AG (2001) The impact of "bursting" thalamic impulses at a neocortical synapse. Nat Neurosci 4:402-408.

Uhlrich DJ, Tamamaki N, Sherman SM (1990) Brainstem control of response modes in neurons of the cat's lateral geniculate nucleus. Proc Natl Acad Sci USA 87:2560-2563.

Uhlrich DJ, Manning KA, Xue J (2002) Effects of activation of the histaminergic tuberomammillary nucleus on visual responses of neurons in the dorsal lateral geniculate nucleus. J Neurosci 22:1098-1107.

Urban I, Richard P (1792) A stereotaxic atlas of the New Zealand rabbit's brain. Springfield, MA: Thomas.

Ursin H, Kaada BR (1960) Subcortical structures mediating the attention response induced by amygdala stimulation. Exp Neurol 2:109-122.

Van Bockstaele EJ, Colago EE, Valentino RJ (1998) Amygdaloid corticotropin-releasing factor targets locus coeruleus dendrites: substrate for the coordination of emotional and cognitive limbs of the stress response. J Neuroendocrinol 10:743-757.

Whalen PJ, Kapp BS, Pascoe JP (1994) Neuronal activity within the nucleus basalis and conditioned neocortical electroencephalographic activation. J Neurosci 14:1623-1633.

Wilensky AE, Schafe GE, LeDoux JE (1999) Functional inactivation of the amygdala before but not after auditory fear conditioning prevents memory formation. J Neurosci 19:RC48(1-5).

Zhu JJ, Lytton WW, Zue JT, Uhlrich DJ (1999) An intrinsic oscillation in interneurons of the rat lateral geniculate nucleus. J Neurophysiol 81:702-711. 